\title{
Johann Gottfried Herder
}




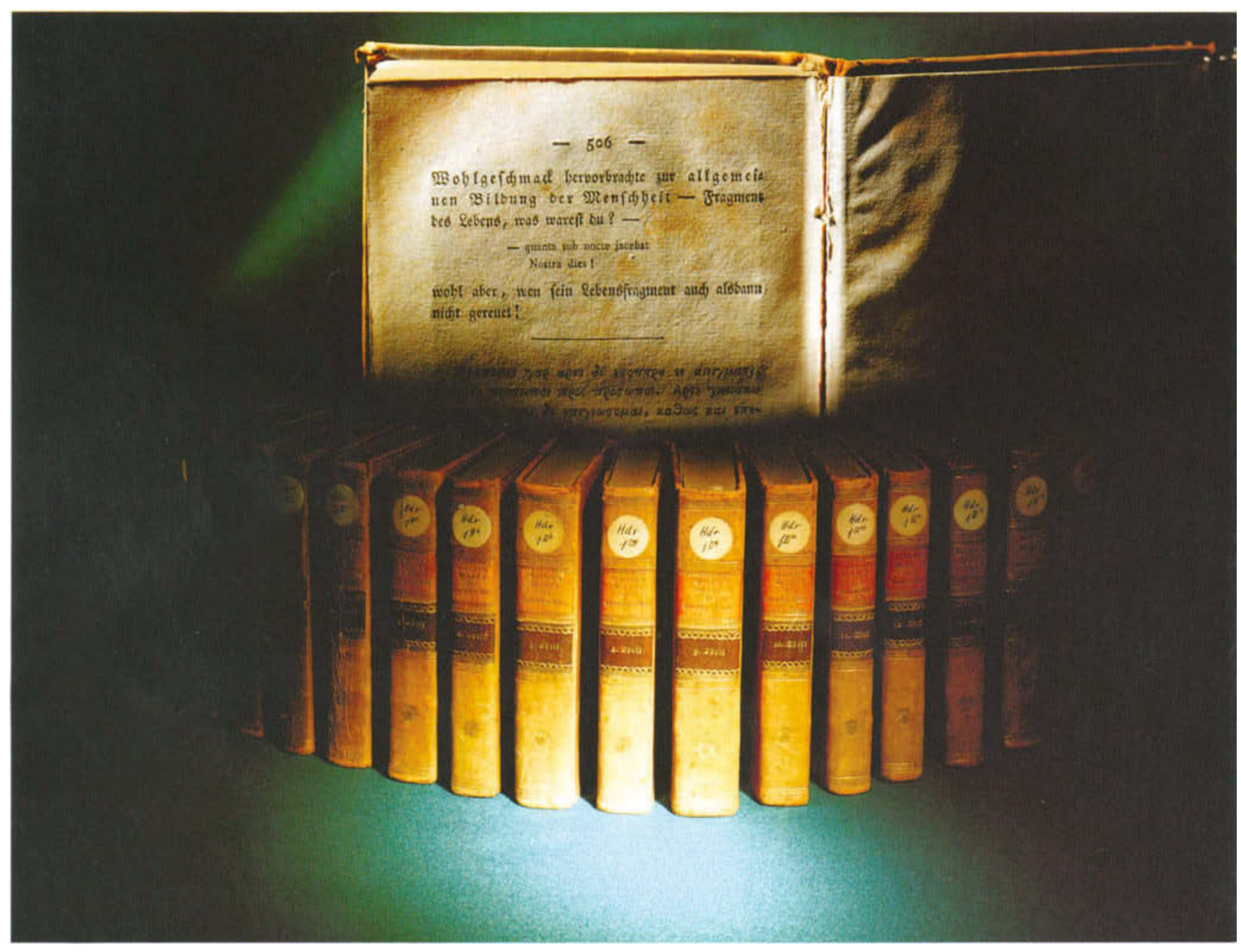




\title{
Johann Gottfried Herder
}

Ahndung künftiger Bestimmung

\author{
Herausgegeben \\ von der Stiftung Weimarer Klassik \\ Goethe-Nationalmuseum
}

1994

Verlag J. B. Metzler

Stuttgart und Weimar 


\section{Edition Weimarer Klassik Band 2}

Erschienen anläßlich der Ausstellung der Stiftung Weimarer Klassik

in Zusammenarbeit mit der Staatsbibliothek zu Berlin - Preußischer Kulturbesitz

zum 250. Geburtstag von Johann Gottfried Herder

Ausstellung und Katalog:

Egon Freitag und Christian Juranek mit Beiträgen von Kurt Wölfel, Renate Müller-Krumbach und Claude Keisch

Ausstellungskonzeption:

Christian Juranek und Renate Müller-Krumbach unter Mitarbeit von Egon Freitag und Regine Otto

Ausstellungseinrichtung: Christian Juranek und Renate Müller-Krumbach

Katalogredaktion: Elke Richter

Ausstellungsaufbau: Lutz Brose, Jürgen Ersfeld, Michael Oertel, Uwe Seeber

Schreibarbeiten: Luba Bens und Barbara Friedrich

Ausstellungsorte:

Kunsthalle am Theaterplatz Weimar

22. Juli bis 26. August 1994

Zentrales Ausstellungsgebäude der Staatlichen Museen Preußischer Kulturbesitz Berlin

16. Dezember 1994 bis 28. Januar 1995

Ahndung künftiger Bestimmung: vgl. Kat.-Nr. 172

Die Deutsche Bibliothek - CIP-Einheitsaufnahme

Johann Gottfried Herder, Ahndung künftiger Bestimmung :

[erschienen anläßlich der Ausstellung der Stiftung Weimarer Klassik in Zusammenarbeit mit der Staatsbibliothek zu Berlin - Preußischer Kulturbesitz zum 250. Geburtstag von Johann Gottfried Herder ; Kunsthalle am Theaterplatz Weimar, 22. Juli bis 26. August 1994 ; Zentrales Ausstellungsgebäude der Staatlichen Museen Preußischer Kulturbesitz Berlin, 16. Dezember 1994 bis 28. Januar 1995] / hrsg. von der Stiftung Weimarer Klassik, Goethe-Nationalmuseum. [Ausstellung und Katalog: Egon Freitag, Christian Juranek; Katalogred.: Elke Richter]. - Stuttgart ; Weimar : Metzler, 1994

(Edition Weimarer Klassik ; Bd. 2)

ISBN 978-3-476-01254-8

ISBN 978-3-476-03559-2 (eBook)

DOI 10.1007/978-3-476-03559-2

NE: Freitag, Egon; Juranek, Christian; [Hrsg.]; Ausstellung zum 250. Geburtstag von Johann Gottfried Herder <1 994-1 995, Weimar; Berlin $>$; Kunsthalle am Theaterplatz $<$ Weimar $>$; Stiftung Weimarer Klassik; GT

(C) 1995 Springer-Verlag GmbH Deutschland Ursprünglich erschienen bei J. B. Metzlersche Verlagsbuchhandlung und Carl Ernst Poeschel Verlag GmbH in Stuttgart 1995 


\section{Inhalt}

Geleitwort zur Ausstellung

Von Renate Müller-Krumbach . . . . . . . . . . . . . . . . . . . . . . . . . . . . . .

"Hundert zerstreute Spiegelscherben zu stellen, daß sie die Strahlen auf einen Punkt werfen" Von Kurt Wölfel . . . . . . . . . . . . . . . . . . . . . . . . . . . . . . . . . . . . .

I. Eigenwelt und Umwelt. Mohrungen, Königsberg, Riga 1744-1769

Von Egon Freitag

II. Welterfahrung und Selbstfindung. Seereise, Frankreich, Darmstadt, Straßburg 1769-1771

Von Christian Juranek

III. Grundlegung und Existenzverbreiterung. Bückeburg 1771-1776

Von Christian Juranek

IV. Enge und Weite. Weimar 1776-1803

Von Egon Freitag . . . . . . . . . . . . . . . . . . . . . . . . . . . . . . . . . . . . .

V. Ahndung künftiger Bestimmung. Miszellen zu einer Rezeptionsgeschichte

Von Christian Juranek

Verzeichnis der Erstdrucke von Herders Werken

Von Christian Juranek

Zeittafel

Von Egon Freitag

Auswahlbibliographie. Mit Siglennachweis

Von Christian Juranek

Personen- und Ortsregister

Von Elke Richter

Photonachweis 


\title{
Fragment des Lebens, WAS WAREST DU?
}

\author{
Johann Gottfried Herder
}




\section{Geleitwort zur Ausstellung}

Herders Texte werden nicht zitiert. Man kennt ihn als wichtigen Theoretiker des 18. Jahrhunderts, als Geschichtsschreiber und Sprachforscher, als Theologen und Nachdichter, aber sein Werk, als Gesamtausgabe von Bernhard Suphan in 33 Bänden in Weimar ediert, wird vorwiegend von Forschung und Wissenschaft benutzt.

Als vierter der Weimarer Klassiker neben Goethe, Schiller und Wieland wurde er schon von den eigenen Zeitgenossen wie selbstverständlich für diese Glanzzeit der deutschen Literatur in Anspruch genommen, sein Denkmal war das erste in der Stadt seines Wirkens.

Der Aufenthalt in Weimar wurde für Johann Gottfried Herder, der auf Betreiben Goethes die Berufung des Herzogs Karl August in das höchste geistliche Amt des Herzogtums annahm, in fast dreißig Jahren die Zeit umfangreichster schöpferischer Produktivität. Ideen, Theorien, hypothetische Untersuchungen, die schon in Riga oder Bückeburg erwogen worden waren, kamen in der Weimarer Superintendentur zur Ausführung, obwohl der Autor sich eher unglücklich als kreativ fühlte.

Ihm, den Goethe "Bruder" nannte, den Wieland hoch schätzte und Schiller respektierte, waren Amt und Aufgaben, unzureichendes Einkommen und wechselnde Gesundheit Last und Beeinträchtigung zugleich, bedeuteten für den Autor Behinderung und Beschränkung, für den Menschen Verbitterung und Enttäuschung. Seine Briefe, deren kommentierte Ausgabe bisher neun Bände umfaßt (herausgegeben von der Stiftung Weimarer Klassik durch Günter Arnold), lesen sich als unmittelbarstes Lebenszeugnis und verdeutlichen Herders faszinierendes Sprachtalent. Hier wird die Annäherung an den Schriftsteller durch das Biographische erleichtert, Bildhaftes ermöglicht den Zugang zum Gedanken.

Ähnliche Prinzipien verfolgen Literaturausstellungen und Literaturmuseen. Um das Bild der Weimarer Klassiker abzurunden, wurden erstmals 1963 sowohl ein Wielandwie auch ein Herdermuseum eingerichtet, das Herdermuseum in der 2. Etage des Kirms-Krackow-Hauses, seinen Wirkungsstätten nahegelegen. Da das Haus wegen unumgänglicher Restaurierungen geschlossen werden mußte und kein angemessner anderer Standort zu finden war, blieb der Wunsch, zum 250. Geburtstag Herders ein neues Museum für ihn zu schaffen, unerfüllbar. Im Gegensatz zu den Wohnhäusern Goethes und Schillers kann das Herderhaus nicht museal genutzt werden, da es in schöner Tradition der Nachfolger im Amt, der jeweilige Superintendent, bewohnt. 
Die Ausstellung, die die Museen der Stiftung Weimarer Klassik konzipierten und gestalteten, ist im Vergleich zum ehemaligen Herdermuseum durch Ankäufe und Leihgaben erweitert worden, thematisch wurden Beispiele zur Wirkungsgeschichte Herders einbezogen. Die Schwierigkeit, Literatur anschaulich zu machen, ist hinlänglich bekannt. Texte, die gelesen werden sollen, sind in einer Vitrine fehl am Platz, - es sei denn, als Gedankenstütze -, Zitate bleiben Ausschnitte und dienen bestenfalls zur Anregung des Selbstlesens. Eine Biographie wie die Goethes, die durch die vielseitige Sammeltätigkeit und verschiedenartige Interessenlage sehr viel Gegenständliches hinterlie8, hat J. G. Herder nicht aufzuweisen. So galt es, seinen allgemein eigentlich kaum bekannten Lebensweg und sein noch weniger gegenwärtiges Werk durch sinnfällige Bezüge sichtbar zu machen.

Die Annäherung an unbekannte Texte versucht die Ausstellung durch die Präsentation der handschriftlichen Fassungen spannungsreicher zu machen. Durch die großzügige Ausleihung der Autographen aus der Staatsbibliothek zu Berlin - Preußischer Kulturbesitz war es möglich, Herders handschriftliche Entwürfe oder Endfassungen in Weimar auszustellen und dem Interessierten die Möglichkeit zu geben, über den Schreibprozeß mit Korrekturen und Verschiebungen sich dem eigentlichen Herdertext zu nähern. Verquickt mit biographischen Bezügen wird in der Ausstellung der Personenkreis aufgebaut, der für das Leben Herders bedeutungsvoll war. Sein Porträt, durch Lebensalter und jeweilige Künstlersicht gewandelt, ist in allen wesentlichen Varianten vorhanden. Die Annäherung an die Biographie wird durch Topographisches, Persönliches und Sachzeugnisse angeboten, die sowohl aus eigenen Beständen stammen als auch als Leihgaben aus dem Freien Deutschen Hochstift Frankfurt, aus der Evangelisch-lutherischen Kirchengemeinde Weimar und von vielen anderen Leihgebern kamen.

Die Wirkungsgeschichte von Herders Leben und Werk, gerade auf dem bildkünstlerischen Sektor noch nicht umfassend erforscht, brachte, in Vorbereitung der Ausstellung, erstaunlich viel Unbekanntes und Spannendes ans Licht. Die Geschichte der Herder-Denkmäler ist ebenso interessant wie die Reaktionen der nachfolgenden Literatengenerationen. Auch die Ausstellung 1994 ist Rezeptionsgeschichte, an ihrem Umgang mit dem Schwierigen wird sie gemessen werden müssen. Herder beschließt sein Charakterbild Immanuel Kants mit den Worten: Der bescheidene Mann aber verschmähet jede prahlende scholastische Inschrift, sein Geist lebt in seinen Werken. 
Für Rat und Unterstützung danken wir besonders:

\section{Berlin:}

Staatsbibliothek zu Berlin - Preußischer Kulturbesitz: Leiter der Handschriftenabt. Herr Dr. Tilo Brandis, Leiter der Kartenabt. Herr Dr. Lothar Zögner; Alte Nationalgalerie zu Berlin - Preußjscher Kulturbesitz: Herr Dr. Bernhard Maaz, Herr Dr. Claude Keisch; Gemäldegalerie der Staatlichen Museen Preußischer Kuturbesitz zu Berlin: Herr Prof. Dr. Henning Bock sowie KaiserFriedrich-Museums-Verein; Wissenschaftskolleg zu Berlin: Herr Prof. Dr. Peter Wapnewski, Herr Prof. Dr. Kurt Wölfel

\section{Bregenz:}

Vorarlberger Landesmuseum: Frau Mag. Jutta Neudorfer

\section{Bückeburg:}

SHD Fürst Philipp Ernst zu Schaumburg-Lippe; Schaumburg-Lippisches Landesmuseum und Schaumburg-Lippischer Heimatverein sowie Frau Dr. Sommer, Bückeburg/Hannover; Schaumburg-Lippische Landeskirche; Niedersächsisches Staatsarchiv in Bückeburg: Herr Dr. Böhme

\section{Darmstadt:}

Hessisches Landesmuseum: Herr Dr. Hans-Dieter Pohl; Hessisches Staatsarchiv Darmstadt: Herr Dr. Jürgen Rainer Wolf

Dresden:

Staatliche Kunstsammlungen Dresden/Kupferstichkabinett: Kustos Herr Glauberecht Friedrich; Herr Werner Kohlert

Düsseldorf:

Anton-und-Katharina-Kippenberg-Stiftung/Goethe-Museum Düsseldorf: Herr Direktor Dr. Volkmar Hansen

Frankfurt am Main:

Freies Deutsches Hochstift/Frankfurter Goethe-Museum: Frau Dr. Petra Maisak, Herr Dr. Jürgen Behrens

Halberstadt:

Das Gleimhaus: Herr Direktor Dr. Horst Scholke

Hamburg:

Museum für Hamburgische Geschichte: Herr Dr. Helmut Glagla, Frau Dr. Gisela Jaacks

Jena:

Hertig \& Schwenzer, Professionelle Phototechnik

Lüneburg:

Ostpreußisches Landesmuseum: Herr Kustos Dr. Barfod; Carl-Schirren-Gesellschaft e. V. /

Das Deutsch-Baltische Kulturwerk: Frau Renate Adolphi

Marbach am Neckar:

Schiller-Nationalmuseum: Herr Dr. Michael Davidis, Frau Margot Pehle

München:

Architekturmuseum der TU München: Prof. Dr.-Ing. W. Nedinger; Bayerische Staatsbibliothek: Frau Dr. Sigrid von Moisy; Bayerische Staatsgemäldesammlung: Frau Landeskonservatorin Dr. Gisela Goldberg, Herr Dr. Christian Zerl; Deutsches Museum: Leiterin der Sondersammlungen und Dokumentationen Frau Dr. Eva A. Mayring; Münchner Stadtmuseum: Herr Oberkonservator Dr. Volker; Staatliche Graphische Sammlung: Frau Dr. Gisela Scheffler; Stadtarchiv München: Herr Archivrat Dr. Schwab, Frau Irmtraud Stockinger; Städtische Galerie im Lenbachhaus: Frau Dr. Barbara Eschenburg; Herr Karl Neuwirth

\section{Münster in Westfalen:}

Universitäts- und Landesbibliothek Münster in Westfalen: Frau Heerdt 


\section{Nürnberg:}

Germanisches Nationalmuseum: Frau Archivdirektorin Dr. Freifrau von Andrian-Werburg, Herr Dr. Rainer Schoch

\section{Oldenburg:}

Landesbibliothek Oldenburg: Herr Direktor Dr. Reindel

\section{Regensburg:}

Museen der Stadt: Herr Konservator Dr. Peter Germann-Bauer

\section{Reinbek bei Hamburg:}

Herr Günther Specovius

\section{Renchen:}

Bürgermeisteramt der Stadt, Herr Bürgermeister Klaus Brodbeck

\section{Riga:}

Historisches Museum Lettland: Frau Anita Meinarte; Museum für die Geschichte Rigas und der Schiffahrt: Frau Direktorin Klara Radzina; Rigaisches Museum für Geschichte der Literatur und der Künste: Herr Direktor P. Zirnitis

\section{Straßburg:}

Stadtarchiv

Tartu (Dorpat)/Estland:

Direktion der Universitätsbibliothek: Frau Direktorin Malle Ermel

\section{Weimar:}

Evang.-luth. Kirchengemeinde Weimar: Herr Superintendent Werner Lässig, Frau Vogel;

Herr Dr. Herbert von Hintzenstern; Herr Restaurator Matthias Krahnstöver; Kunstsammlungen zu Weimar: Herr Direktor Dr. Rolf Bothe, Frau Dr. Bestgen, Frau Kustodin Helga Hoffmann, Herr Kustos Dr. Vogelsang, Herr Katzer; Thüringisches Landesamt für Archäologische Denkmalpflege: Herr Restaurator Leithner

Wörlitz:

Staatliche Schlösser und Gärten Wörlitz, Oranienbaum, Luisium: Abteilungsleiter Forschung und Publikation Herr Uwe Quilitzsch

Wolfenbüttel:

Herzog August Bibliothek

\section{Stiftung Weimarer Klassik:}

Herr Dr. Günter Arnold, Frau Luba Bens, Herr Lutz Brose, Frau Petra Ellermann-Minda, Frau Angelika Emmrich, Herr Jürgen Ersfeld, Frau Barbara Friedrich, Frau Sigrid Geske, Frau Viola Geyersbach, Herr Dr. Jochen Golz, Frau Katrin Gränz, Frau Beate Grüneberger, Herr Dr. Michael Knoche, Frau Dr. Katharina Krügel, Frau Katrin Lehmann, Frau Gisela Nebiger, Herr Michael Oertel, Frau Margarete Oppel, Frau Gabriele Oswald, Frau Dr. Regine Otto, Frau Katharina Popov, Frau Susanne Schroeder, Frau Inga Schulz, Herr Dr. Gerhard Schuster, Herr Uwe Seeber, Herr Robert Steiner, Frau Dr. Roswitha Wollkopf. 